\title{
Quaderni
}

QUADERN I Communication, technologies, pouvoir

\section{Idéologies et hégémonie : la classe dirigeante au prisme des think tanks}

\section{Geoffrey Geuens}

\section{(2) OpenEdition}

1 Journals

\section{Édition électronique}

URL : http://journals.openedition.org/quaderni/510

DOI : 10.4000/quaderni.510

ISSN : 2105-2956

\section{Éditeur}

Les éditions de la Maison des sciences de l'Homme

\section{Édition imprimée}

Date de publication : 5 octobre 2009

Pagination : 69-77

\section{Référence électronique}

Geoffrey Geuens, «Idéologies et hégémonie : la classe dirigeante au prisme des think tanks », Quaderni [En ligne], 70 | Automne 2009, mis en ligne le 05 octobre 2011, consulté le 20 avril 2019. URL : http://journals.openedition.org/quaderni/510; DOI : 10.4000/quaderni.510 


\section{$D$ ossier}

\section{idéologies et hégémonie : la classe dirigeante au prisme des think tanks}

\section{Geoffrey Geuens}

Chargé de cours, département des Arts et Sciences de la Communication, Université de Liège
La problématique des think tanks est directement liée à l'idée de "société civile" laquelle formule - tenant de l'effet de mode autant que d'imposition - a su s'imposer, au fil du temps, comme l'un des éléments centraux des discours néo-libéraux et technocratiques, incarnant tantôt la résistance légitime à un État totalitaire confisquant le débat public, tantôt l'éclosion d'un véritable contre-pouvoir citoyen annonçant l'avènement de la démocratie participative. Un nouveau sens commun, en phase avec la société de la gouvernance, s'est ainsi imposé et avec lui une série d'oppositions - aussi stériles que banales - dont personne, sauf à de rares exceptions, ne trouve légitime d'interroger le bien-fondé et d'évaluer le poids de réalité.

La dichotomie de la société civile et de l'État est assurément l'une des plus tenaces et des mieux partagées, la montée en puissance de l'une annonçant en quelque sorte le retrait programmé - sinon même la disparition - du second. Dans cette perspective, qu'on se représente le monde des think tanks sous les traits - toujours forcés - d'agences intellectuelles instrumentalisées par le monde des affaires et de la politique ou, au contraire, d'institutions de réflexion jouissant d'une pleine autonomie, les termes (influence, immixtion, indépendance) se renforcent mutuellement et, avec eux, l'implicite dont ils sont porteurs : celui de l'extériorité réciproque de la société civile et du pouvoir. De distinction méthodologique dans l'analyse, la dissociation en question se voit alors métamorphosée, in fine, en dissociation de principe sinon même de nature.

L'étude scientifique des relations d'interpéné- 
tration entre État, marché et institutions idéologiques ne peut cependant - sauf à risquer de manquer son objet - faire l'économie d'une réflexion spécifique portant surl'univers des think tanks entendu comme sous-espace constitutif du champ du pouvoir, dont l'imbrication croissante aux sphères médiatiques, financières et politiques s'exprimerait et se consoliderait à travers la circulation ininterrompue de certains agents sociaux. C'est pour cette raison que l'on optera, quant à nous, pour une démarche de type sociobiographique permettant précisément, à suivre Dezalay et Garth, le dépassement des clivages artificiels introduits par «les découpages institutionnels ou disciplinaires, qui occultent tout le jeu de relations personnelles entre les différents univers sociaux. $»^{1}$. On fera ainsi de la dissociation entre théories régionales (économie, politique, idéologie) une distinction strictement pratique et non normative, cette forme implicite de censure n'ayant pour seul véritable fondement que celui d'une division essentiellement académique du travail de recherche. Les personnalités américaines et françaises évoquées dans les pages qui suivent - dirigeants et conseillers de boîtes à idées - ne seront donc pas appréhendées dans leur singularité propre mais en tant qu'elles sont autant de formes incarnées de relations sociales génériques entre monde des affaires, pouvoir d'État et think tanks. C'est que la logique d'entrelacement des univers sociaux considérés a toujours besoin de s'incarner dans des individus en chair et en os - à la fois singuliers et interchangeables - pour s'accomplir pleinement.

Si ces situations de multipositionnalité sont au principe d'une formidable accumulation individuelle de capitaux sociaux et participent à la construction de réseaux de sociabilité indispensables à l'exercice effectif du pouvoir, il convient surtout de noter que cette dynamique sociale - celle du cumul - garantit également l'intégration, à tout le moins relative, des diverses fractions dirigeantes. On sera ainsi particulièrement attentif au rôle joué dans ce cadre par certains responsables se signalant par la multiplicité de leurs positions institutionnelles. Agissant en quelque sorte comme les représentants de la fraction hégémonique du bloc au pouvoir, ces agents sociaux préservent - de façon plus ou moins sentie et consentie - les intérêts globaux des dominants par rapport aux profits parcellaires et multiples associés à chaque univers professionnel.

\section{L'hégémonie des think tanks mainstream aux États-Unis}

Il est tout d'abord intéressant de constater que les conseils d'administration des principaux groupes médiatiques américains (Time Warner, Disney, News Corporation, General Electric, Viacom $)^{2}$ ne sont pas seulement bipartisans d'un strict point de vue politique, accueillant côte à côte démocrates et républicains. Ils sont également mainstream sur le plan idéologique, les dirigeants de ces entreprises se rattachant, pour l'essentiel, à des think tanks qualifiés par G. William Domhoff de modérément conservateurs (« moderate-conservative »). Qu'il s'agisse du Council on Foreign Relations (CFR), de l'Aspen Institute ou de la Brookings Institution - trois boîtes à idées fortement intégrées à l'industrie de la communication - ces groupes de réflexion ont été successivement unilatéralistes et multi- 
latéralistes, libre-échangistes et protectionnistes, anti-étatistes et interventionnistes. À l'image de la grande bourgeoisie d'affaires éclairée, ils se reconnaissent simultanément dans le libéralisme économique et certaines politiques de régulation, dans la défense de la compétitivité des entreprises nationales et les vertus de la redistribution sociale, et cela au gré de leurs intérêts et des rapports de force du moment.

On constatera à l'inverse que les responsables de think tanks affiliés à l'aile progressiste du Parti démocrate (Economic Policy Institute, Center on Budget and Policy Priorities) ou à la mouvance néo-conservatrice (American Enterprise Institute, Hoover Institution, Manhattan Institute) ne sont, quant à eux, que rarement cooptés dans les conseils d'administration des plus puissantes entreprises médiatiques des États-Unis. La très faible représentativité d'organisations aussi réputées que le Cato Institute, l'Heritage Foundation ou encore l'Hudson Institute, est à cet égard révélatrice de ce que ces structures de la droite radicale américaine et leurs mécènes (Douglas Allison, Holly Coors, Richard Mellon Scaife) ne sont probablement pas en mesure de représenter les intérêts à long terme du bloc au pouvoir. Le rôle primordial que ces boîtes à idées ont joué en leur temps dans l'élaboration, la réalisation et la justification des politiques entreprises par les administrations Reagan et Bush est certes indiscutable ; mais cela ne signifie pas pour autant que le monde des affaires - et, plus particulièrement, la haute finance - soit passé, aux États-Unis et en une petite vingtaine d'années seulement, de la défense d'un keynésianisme modéré à celle d'une économie parfaitement dérégulée. Tout au plus, ce rappel historique est-il l'indice de ce que le capitalisme réel entretient un rapport purement instrumental à l'idéologie de marché.

Dotés, au contraire, d'une très forte centralité dans le champ du pouvoir, des think tanks tels que le CFR ou Brookings constituent quant à eux l'une des composantes de ce que Michael Useem nomme l' «Inner Circle $»^{3}$, ce noyau dur des agents et institutions de la grande bourgeoisie d'affaires dont les membres sont sociologiquement disposés à transcender les profits sectoriels au bénéfice de l'intérêt collectif des dominants et à sacrifier le libéralisme doctrinal au « libéralisme des oligopoles ». Le pouvoir allant au pouvoir, on ne sera dès lors pas surpris de constater que ces mêmes boîtes à idées sont de celles qui tirent avantage de relais au plus haut niveau de l'appareil d'État, bénéficiant en outre du soutien des principaux groupes industriels et financiers nationaux. Ainsi, selon G. William Domhoff, «vingt-cinq des trente-trois administrateurs [de Brookings] détiennent cinquante mandants dans quarante-huit entreprises [parmi lesquelles] Alcoa, AT\& T, Coca-Cola, CSX, Dow Chemical, et Levi Strauss $»^{4}$, et cela alors même qu'aucun administrateur de la Fondation Heritage ne siège dans une entreprise du Who Knows Who.

Aux États-Unis, ce sont bien par conséquent les membres de cette élite industrielle et financière rassemblant "républicains de gauche» et «démocrates de droite»- pour reprendre l'expression du Chancelier Schmidt ${ }^{5}$ au sujet des dirigeants du CFR - qui assurent une relative convergence idéologique entre les principales boîtes à idées du pays. Dotés d'un don d'ubiquité singulièrement développé, ils accumulent les positions d'administrateur dans les plus grandes 
entreprises américaines et les postes à responsabilité dans les principaux think tanks, se jouant aussi bien des frontières thématiques que des antagonismes politiques traditionnels. Le listing des mandats détenus par certains conseillers du CFR est emblématique de cette multiplicité de positions institutionnelles.

Les deux co-présidents du CFR ne dérogent évidemment pas à la règle. La républicaine Carla Hills, ancienne représentante au Commerce des États-Unis (1989-1993), a été administratrice de Time Warner, Lucent, ChevronTexaco, AT\&T, IBM et American Airlines. Membre des comités consultatifs internationaux de J.P. Morgan Chase, AIG et Coca-Cola, elle est présidente de 1'Inter-American Dialogue, co-présidente du conseil international du Center for Strategic and International Studies (CSIS), membre du comité exécutif de l'Institute for International Economics, de la Commission trilatérale et administratrice de l'International Crisis Group (ICG). Quant au démocrate Robert Rubin, ex-Secrétaire au Trésor (1995-1999) devenu conseiller économique de Barack Obama, il est aujourd'hui vice-président de Citigroup et administrateur de Ford Motor. Membre de la Commission trilatérale, il fut à l'origine de l'Hamilton Project, un mini-think tank de politique économique affilié à la Brookings Institution, dont sont issus certains conseillers de l'actuel président des États-Unis (Austan Goolsbee, Laura Tyson).

Quant aux groupes de réflexion globaux tel que la Commission trilatérale - là où s'expriment, selon Stephen Gill, les intérêts des éléments «les plus dynamiques du capital transnational $\gg^{6}-\mathrm{ils}$ réunissent essentiellement les représentants de firmes américaines (Time Warner, Disney, News Corp.), européennes (Bertelsmann, Prisa, Reuters) et japonaises (Sony) particulièrement actives à l'étranger.

\section{Le marché des idées en France : concurrence, collaboration, concentration}

En France, les think tanks mainstream - modérément conservateurs et bipartisans - représentent également près de la moitié de l'ensemble des boîtes à idées auxquelles l'industrie nationale de la communication se voit le plus systématiquement imbriquée. Une organisation telle que l'Institut français des Relations internationales (Ifri) met ainsi en contact près de trois quarts des entreprises médiatiques de l'Hexagone, ce que fit également en son temps la Fondation Saint-Simon. Quant aux groupes de réflexion néo-libéraux - généraliste (Institut Montaigne), patronaux (Institut de l'Entreprise) ou chargés de la refondation de l'UMP (Fondation pour l'Innovation politique/FONDAPOL) - et autres think tanks associés au renouveau social-démocrate, ils viennent compléter l'offre d'expertise sur le marché national des idées. Cette représentation toute à la fois pluraliste et hétéroclite de l'industrie française des think tanks demande néanmoins à être interrogée à la lumière d'un double constat.

Il faut noter, dans un premier temps, que les entreprises de presse traditionnelles ne sont généralement associées qu'à une poignée de boîtes à idées clairement partisanes sur le plan politique, ce qui est le cas du Figaro (FONDAPOL, Fondation Concorde) ou, à l'opposé, du Monde et de Libération dont les dirigeants sont 
plutôt eux affiliés à des groupes de réflexion de centre-gauche, tendance « troisième voie» $(\mathrm{La}$ République des Idées, En Temps réel). En réalité, seuls les conglomérats actifs dans le secteur de la communication (Suez, Vivendi, Lagardère) se voient reliés à un large éventail idéologique de think tanks. La présence dans chacun de ces groupes industriels de dirigeants affiliés à des organisations néo-progressistes, des institutions patronales et/ou des boîtes à idées mainstream n'est pas nécessairement le signe de ce que ces firmes françaises - et leur filiales «médias » - seraient parfaitement pluralistes, se faisant indirectement l'écho des divers courants de pensée existants. Cette recherche d'unité dans la diversité semble plutôt témoigner du fait que certaines contradictions secondaires - internes au groupe social dirigeant - n'excluent nullement l'existence d'un accord de fond, au moins tacite, sur certaines valeurs et projets politiques. D'autres facteurs participent également à l'édification d'un complexe idéologique à la française relativement intégré.

On pensera tout d'abord au rôle croissant joué par les partenaires financiers des think tanks, qu'il s'agisse de généreux donateurs privés, d'institutions publiques (État français, Commission européenne, OTAN) ou de puissantes sociétés hexagonales. Soutenant massivement les principaux groupes de réflexion patronaux, ces entreprises du CAC40 n'en négligent pas pour autant les institutions mainstream consacrées aux questions de politique étrangère et les boîtes à idées se reconnaissant dans ce qu'il est désormais convenu d'appeler la « troisième voie ». Le leader mondial de l'assurance Axa apporte ainsi sa contribution à droite, à l'Institut Montaigne et
l'Institut de l'Entreprise (IDEP), au centre-gauche à Confrontations Europe, mais aussi à l'Ifri, think tank mainstream par excellence. Ce n'est évidemment pas là le seul exemple du genre : la BNP Paribas finance l'Institut Montaigne, Entreprise et Progrès, l'Ifri, Aspen France et Confrontations Europe; le groupe énergétique Suez est partenaire de l'Institut Montaigne, l'Ifri, Confrontations Europe et Aspen France; Total soutient à son tour l'Institut Montaigne, la Fondation pour la Recherche stratégique, Entreprise et Progrès, l'Ifri, Confrontations Europe et Aspen France ; quant à Schneider Electric, il contribue au budget de l'Institut Montaigne, l'IDEP, l'Ifri et Aspen France.

Il convient aussi d'être attentif aux multiples partenariats s'instaurant entre des boîtes à idées théoriquement concurrentes dans le champ de l'expertise privée. Précisons d'emblée que ces collaborations mettent essentiellement en jeu des think tanks occupant des positions relativement centrales dans le champ du pouvoir, se signalant par une disposition politique au consensus - dissension bien plus que dissidence - et par des prises de position publiques évitant toute forme de radicalité rhétorique. Le noyau dur de l'industrie française des groupes de réflexion réunit ainsi à son enseigne un ensemble d'organisations allant « de la droite intelligente à la gauche intelligente $»^{7}$, selon l'expression chargée d'Alain Minc évoquant, en son temps, les dirigeants de la Fondation Saint-Simon. Quant aux boîtes à idées plus ouvertement militantes - qu'elles relèvent de la droite ultra-libérale (Institut Turgot) ou de la gauche altermondialiste (Fondation Copernic) - elles se voient, en quelque sorte, renvoyées à la périphérie du système idéologique national. Le 
domaine des études européennes est à cet égard particulièrement emblématique : Confrontations Europe déclare parmi ses nombreux partenaires institutionnels la Fondation Robert-Schuman et Europartenaires, Europartenaires précise lui entretenir des relations suivies avec Confrontations Europe, l'Ifri et Notre Europe, quant aux Amis de l'Europe, ils sont eux associés à la Fondation Robert-Schuman, Notre Europe et Confrontations Europe. La boucle est bouclée.

Quant aux administrateurs multiples issus pour la plupart du secteur financier, de la grande industrie et de la haute fonction publique, ils peuvent être légitimement considérés - pour parler le langage un peu oublié d'un Gramsci - comme les intellectuels organiques de la fraction hégémonique du bloc au pouvoir. Qu'ils soient les héritiers du « socialisme de la rigueur » - fidèle (Gérard Mestrallet) ou nouvellement converti au MoDem (Jean Peyrelevade) - d'anciens partisans convaincus de l'UDF (Michel Pébereau) ou de nouveaux alliés de 1'UMP et du Président Sarkozy (Claude Bébéar, Francis Mer), ces figures éminentes du monde des affaires représentent autant de formes incarnées de relations sociales génériques entre économie, pouvoir d'État et think tanks (voir tableau en annexe).

On l'aura compris, aux États-Unis comme en France, la classe dirigeante peut ainsi se reconnaître dans un certain nombre de courants de pensée - néolibéralisme doctrinal pour la droite classique, socialisme libéral pour la gauche de pouvoir - qui, relativement divergents les uns des autres, n'en sont pas moins tous compatibles avec les exigences minimales de l'accumulation du capital, de la défense de la compétitivité des entreprises nationales et de la reproduction des rapports sociaux dominants. Se jouant des contradictions politiques et idéologiques secondaires, les représentants de la grande bourgeoisie d'affaires entretiennent par conséquent un rapport purement instrumental au libéralisme, leur pragmatisme s'accommodant fort bien d'un discours social en appelant à une certaine forme de régulation par les pouvoirs publics. Dans cette perspective, la dénonciation convenue de l'industrie des think tanks comme relais privilégié de la seule idéologie libérale fait l'impasse sur le rôle de ces institutions dans la construction, la reproduction et la diffusion d'un discours hégémonique - celui du capitalisme - lequel emprunte autant à l'idolâtrie de marché qu'au fétichisme d'État. 


\section{Gérard MESTRALLET}

Ancien conseiller technique de Jacques Delors au ministère de l'Économie et des Finances (1982-1984), il est actuellement PDG de GDF Suez, administrateur de Saint-Gobain et d'Axa. Membre du comité de parrainage de Confrontations Europe, il siège également au conseil de surveillance d'Aspen France et au bureau exécutif de l'Institut de l'Entreprise.

\section{Jean PEYRELEVADE}

Ex-directeur adjoint du cabinet du Premier ministre Pierre Mauroy et président du Crédit lyonnais, il est aujourd'hui banquier d'affaires chez Leonardo France, administrateur de Bouygues, CMA-CGM, KLM et vice-président du MoDem. Ancien conseiller d'Air liquide, Axa, Club Med, L'Oréal, Lagardère, LVMH, Société générale et Suez, il est aussi passé par les comités consultatifs internationaux d'Allianz et de Power Corporation of Canada. Au centre-gauche, il est administrateur et membre du conseil de direction de Confrontations Europe, membre du comité d'orientation scientifique de la Fondation JeanJaurès, de Terra Nova et du Centre international Pierre Mendès-France, ainsi que trésorier de la République des Idées. Conférencier régulier d'Entreprise et Progrès, Jean Peyrelevade est administrateur de l'Ifri.

\section{Michel PÉBEREAU}

Ancien directeur de cabinet de René Monory au ministère de l'Économie (1978-1980) et actuel président de BNP Paribas, il est également administrateur de Total, Saint-Gobain, Lafarge, Axa et EADS. Président de l'Institut de l'Entreprise et d'Aspen France, il siège en outre au conseil de direction de Confrontations Europe.

\section{Bertrand COLLOMB}

Ex-conseiller technique de René Haby au ministère de l'Éducation nationale (1974-1975), le président d'honneur de Lafarge siège au conseil d'administration de Total, Vivendi Universal, Unilever, ATCO et DuPont ainsi qu'au comité consultatif international d'Allianz. Président de l'AFEP et de l'Ifri, il est aussi président d'honneur de l'Institut de l'Entreprise et membre du comité de parrainage de Confrontations Europe.

\section{Claude BÉBÉAR}

Président d'Axa, il est passé par les conseils d'administration d'Aventis, BNP Paribas, Casino, Saint-Gobain, Vivendi Universal, Schneider Electric et de la Société générale. À droite, il est notamment président de l'Institut Montaigne. Conférencier régulier de l'Ifri et d'Entreprises et Cité, il est membre du comité de soutien du Centre d'Analyse et de Proposition pour l'Afrique (CAPAfrique), un think tank présidé par le Lionel Zinsou. Ce dernier, ex-conseiller de Fabius devenu associé-gérant de la Banque Rothschild, est administrateur de Telos, la boîte à idées progressiste dirigée par Zaki Laïdi.

\section{Francis MER}

Ex-coprésident d'Arcelor et ministre de l'Économie, des Finances et de l'Industrie (2002-2004), il est actuellement président du groupe Safran, administrateur de Rhodia, Adecco et Vale Inco 
(Canada), membre du comité européen de la Deutsche Bank, de Bridgepoint Capital et du conseil international de Marsh \& McLennan (États-Unis). Membre du conseil de surveillance et ancien président de la Fondation pour l'Innovation politique, Francis Mer siège en outre au conseil de direction de Confrontations Europe.
$\mathrm{N}$

1. Yves Dezalay et Bryant G. Garth, La mondialisation des guerres de palais. La restructuration du pouvoir d'État en Amérique latine, entre notables du droit et "Chicago Boys », Paris, Seuil, 2002, p. 36. Les deux auteurs précisent: «Les biographies révèlent les multiples liens qui existent entre des espaces sociaux définis par des catégories institutionnelles ayant précisément pour objet de masquer ce jeu de relations. Les recherches qui considèrent comme allant de soi les cloisonnements construits entre différentes professions ou institutions contribuent à occulter tout le travail de définition et de légitimation inscrit dans cette histoire professionnelle ou institutionnelle. » p. 37.

2. La contribution qui suit s'appuie pour l'essentiel sur une enquête sociographique réalisée dans le cadre d'une thèse de doctorat intitulée Globalisation, communication et champ du pouvoir. Une socio-économie comparée des structures actionnariales et dirigeantes des groupes de médias et des entreprises des NTIC en Europe et aux États-Unis (Université de Liège, 2007). Plus de 2000 dirigeants ayant siégé, entre 2002 et 2006, dans les conseils d'administration des principales entreprises médiatiques et technologiques américaines et européennes ont ainsi fait l'objet d'une étude sociographique. Cette analyse a reposé sur l'exploitation systématique des informations rassemblées à propos de ces dirigeants ; les informations en question concernant leurs positions institutionnelles nationales et internationales - passées ou présentes - occupées notamment dans les champs économique, politique et idéologique.

3. Michael Useem, The Inner Circle. Large Corporations and the Rise of Business Political Activity in the U.S. and U.K., Oxford, Oxford University Press, 1984. 
4. G. William Domhoff, Who Rules America? Power and Politics, McGraw-Hill, 2002, p. 80.

5. Helmut Schmidt, Des puissances et des hommes, Paris, Plon, 1987, pp. 245-246.

6. Stephen Gill, American Hegemony and Trilateral Commission, Cambridge, Cambridge University Press, 1990, p. 9.

7. Laurent Bonelli, Le passé d'une fondation, Mémoire de DEA de Politique comparée et Sociologie politique, Nanterre Paris X, 1999, p. 15.
$R \cdot E \cdot S \cdot U \cdot M \cdot E$

Cet article se propose de repenser l'univers des think tanks comme sous-espace constitutif du champ du pouvoir, dont l'imbrication croissante aux sphères médiatiques, économiques et politiques s'exprimerait et se consoliderait à travers la circulation ininterrompue de certains agents sociaux dominants. Se jouant des contradictions politiques et idéologiques secondaires, ces représentants de la grande bourgeoisie d'affaires entretiennent ainsi un rapport purement instrumental au libéralisme, leur pragmatisme s'accommodant fort bien d'un discours social en appelant à une certaine forme de régulation par les pouvoirs publics. Dans cette perspective, la dénonciation des think tanks comme relais privilégié de l'idéologie libérale fait l'impasse sur le rôle de ces institutions dans la construction, la reproduction et la diffusion d'un discours hégémonique - celui du capitalisme - lequel emprunte autant à l'idolâtrie de marché qu'au fétichisme d'État.

This article seeks to rethink the world of think tanks as a sub-space constituting the field of power; growing links of this sub-space with media industry, economy and politics being expressed and consolidated through the seamless movement of certain social agents. These representatives of the financial bourgeoisie have in fact a purely instrumental relation with liberalism, their pragmatism being well accommodated with a social discourse of public regulation. In this perspective, the denunciation of think tanks as a preferred vehicle for liberal ideology ignores the role of think tanks in the construction, reproduction and dissemination of a hegemonic discourse - that of capitalism - which uses both market idolatry and State fetishism. 
